\title{
Neoadjuvant radiotherapy for resectable hepatocellular carcinoma with portal vein tumor thrombus: a systematic review
}

\author{
Zhewen Wei, Jianjun Zhao, Xinyu Bi, Yefan Zhang, Jianguo Zhou, Zhiyu Li, Zhen Huang, Hong Zhao, \\ Jianqiang Cai \\ Department of Hepatobiliary Surgery, National Cancer Center/National Clinical Research Center for Cancer/Cancer Hospital, Chinese Academy of \\ Medical Sciences and Peking Union Medical College, Beijing, China \\ Contributions: (I) Conception and design: J Cai, H Zhao, Z Wei; (II) Administrative support: J Cai, H Zhao; (III) Provision of study materials or \\ patients: J Zhao, Y Zhang; (IV) Collection and assembly of data: Z Wei, J Zhou, Z Li; (V) Data analysis and interpretation: Z Wei, X Bi, Z Huang; (VI) \\ Manuscript writing: All authors; (VII) Final approval of manuscript: All authors. \\ Correspondence to: Hong Zhao, MD; Jianqiang Cai, MD. Department of Hepatobiliary Surgery, National Cancer Center/National Clinical Research \\ Center for Cancer/Cancer Hospital, Chinese Academy of Medical Sciences and Peking Union Medical College, No. 17 Nanli, Panjiayuan, Chaoyang \\ District, Beijing 100021, China. Email: zhaohong@cicams.ac.cn; caijianqiang188@sina.com.
}

Background: The prognosis of hepatocellular carcinoma (HCC) with portal vein tumor thrombus (PVTT) is extremely poor. The clinical outcome of preoperative radiotherapy (RT) is still controversial. This study aimed to compare the clinical outcomes of combined neoadjuvant RT and hepatectomy with hepatectomy alone for HCC with PVTT.

Methods: Comprehensive database searches were performed in PubMed, the Cochrane Library, EMBASE, and the Web of Science to retrieve studies published from the database creation to July 1, 2020. Only comparative studies that measured survival between neoadjuvant RT followed by hepatectomy and hepatectomy alone were included. The characteristics of the included studies and patients were extracted, and the included data are presented as relative ratio (RR) estimates with $95 \%$ confidence intervals (CIs) for all outcomes. The RRs of each study were pooled using a fixed or random effects model with Review Manager (the Cochrane Collaboration, Oxford, UK) version 5.3. The response rate to RT and the overall survival (OS) rate in neoadjuvant RT followed by hepatectomy and hepatectomy alone were measured.

Results: One randomized and two non-randomized controlled trials with 302 patients were included. Most patients were classified as Child-Pugh A, and Type II and III PVTT were the most common types. After RT, 29 (22.8\%) patients were evaluated as partial response (PR) and had a positive RT response, but nine $(7.1 \%)$ had progressive disease (PD). Neoadjuvant RT followed by hepatectomy was received by 127 $(42.1 \%)$ patients after excluding $15(5.0 \%)$ patients with severe complications or PD after RT, and 160 $(53.0 \%)$ patients received hepatectomy alone. In the randomized controlled trial (RCT), the 1-year OS rate in the neoadjuvant RT group and the surgery alone group was $75.2 \%$ and $43.1 \%$, respectively $(\mathrm{P}<0.001)$. In the two non-randomized studies, a meta-analysis with a fixed effects model showed a longer OS in patients undergoing neoadjuvant RT followed by hepatectomy compared with hepatectomy alone at 1-year follow-up ( $\mathrm{RR}=2.02 ;$ 95\% CI: 1.45-2.80; $\mathrm{P}<0.0001)$.

Conclusions: This systematic review showed that neoadjuvant RT followed by hepatectomy in patients with resectable HCC and PVTT was associated with a longer OS than patients who received hepatectomy alone.

Keywords: Hepatocellular carcinoma (HCC); portal vein tumor thrombosis; neoadjuvant radiotherapy; hepatectomy 
Submitted Dec 20, 2020. Accepted for publication Apr 23, 2021.

doi: $10.21037 /$ hbsn-20-854

View this article at: http://dx.doi.org/10.21037/hbsn-20-854

\section{Introduction}

Liver cancer is estimated to rank sixth among newly diagnosed cancers and fourth in causes of cancer-specific deaths worldwide, with over 8.4 million new cases and 7.8 million deaths annually (1). Hepatocellular carcinoma (HCC) is one of the most common types of liver cancer, with a high recurrence and poor prognosis (2). Portal vein tumor thrombosis (PVTT), which is caused by the tumor invading the portal venous system, commonly occurs in advanced HCC with an extremely poor prognosis, and sorafenib is recommended as the only treatment in Western guidelines $(3,4)$.

Currently, a variety of local treatments are widely applied for HCC patients with PVTT in Asian countries. Transarterial chemoembolization (TACE) is the most common locoregional therapy option for patients with unresectable HCC and PVTT (5). TACE provides a better survival outcome than other supportive treatments for patients with advanced HCC and PVTT who have an adequate liver function (6). Hepatic infusion chemotherapy (HAIC) is another effective local treatment. A systematic review demonstrated that compared to sorafenib, HAIC is associated with higher overall survival (OS) and progression-free survival (PFS), especially for HCC with Type II and III PVTT (7). Similarly, hepatic resection is a potentially curative modality for those suitable for resection who have Type II and III PVTT (8). With the advances in surgical techniques, surgical resection for HCC with PVTT has become an effective and safe method (9). Compared to TACE, hepatic resection demonstrates a longer OS for HCC with Type I and II PVTT $(10,11)$.

Several studies of surgery-based comprehensive treatment such as surgery after radiotherapy (RT) for HCC. RT, which makes unresectable HCC eligible for surgical resection, combined with surgery, may improve these patients' OS $(12,13)$. Also, RT has emerged as an effective modality for HCC with PVTT $(14,15)$. It reportedly reduced the extent of PVTT, with a 2-year OS rate of $67 \%$ for patients who received preoperative RT (16). However, all of these studies of HCC with PVTT are limited by relatively small number of patients and the lack of strong evidence.
We examined the evidence that preoperative RT in HCC with PVTT may improve survival. Here, we carried out a systematic review of all randomized and non-randomized controlled trials of preoperative RT in HCC with PVTT. We present the following article in accordance with the PRISMA reporting checklist (available at https://hbsn. amegroups.com/article/view/10.21037/hbsn-20-854/rc).

\section{Methods}

\section{Literature search}

In this systematic review, we searched PubMed, the Cochrane Library, EMBASE, and the Web of Science to retrieve studies published from the time of the database creation to July 1, 2020. A computer-assisted search was conducted using the following combination of medical subject heading terms (MeSH): ("hepatocellular carcinoma" or "liver cancer" or "HCC") AND ("portal vein tumor thrombus" or "macroscopic vascular invasion" or "PVTT") AND ("liver resection" or "hepatectomy" or "hepatic resection" or "surgical resection") AND ("preoperative or neoadjuvant radiotherapy"). The study protocol is registered in PROSPERO (CRD42020199572).

\section{Study selection}

After the initial retrieval, two authors of us (ZWW and JJZ) independently screened the articles' titles and abstracts to identify potentially relevant studies. Then, the fulltext articles were screened and reviewed independently by the two authors according to the inclusion and exclusion criteria. Disagreements were resolved by a third author (HZ).

\section{Eligibility criteria}

Inclusion criteria: (I) HCC patients (aged between 18 and 70 years) with various types of PVTT who underwent hepatic resection with or without neoadjuvant RT; (II) a comparison of OS or disease-free survival (DFS) or PFS; (III) the median follow-up period was more than 6 months.

Exclusion criteria: (I) patients with a previous history 
of other malignancy and antitumor treatment in the past five years; (II) HCC patients with PVTT who received other treatments instead of preoperative RT alone; (III) conference abstracts, reviews, case reports, letters, editorials, comments, or any studies other than a peerreviewed original research article; (IV) duplicated studies or replicated data reported by the same center or hospital; when studies of duplicate populations were identified, we selected the study that included more institutions or more patients.

\section{Quality assessment}

The randomized controlled trial (RCT) data quality was assessed using Cochrane collaboration tool for assessing the risk of bias. The quality of non-randomized studies was assessed using the Newcastle-Ottawa Scale. The study quality was judged to be high if the Newcastle-Ottawa Scale score was at least 7 points (out of a possible 9 points) and categorized as good ( 3 or 4 points in the selection domain, 2 or 3 points in the exposure and outcome domain, and 1 or 2 points in the comparability domain). Otherwise, study quality was considered low.

\section{Data extraction}

One author (ZWW) independently extracted the following data from all included studies: authors, country, study design, neoadjuvant RT, patient characteristics, therapeutic effects of RT, and clinical outcomes of surgery. Data such as relative ratios (RRs) and $95 \%$ confidence intervals (CIs) of OS were calculated. These data were checked by a second author (XYB). If there was disputed data, a third author (JQC) was invited to participate in the discussion to resolve disagreements and reach a consensus.

\section{Statistical analysis}

The outcomes included OS rates. OS rates were assessed for different years, with some data being obtained from survival curves. The included data are presented as RR estimates with $95 \%$ CIs for all outcomes. The RRs of each study were pooled using a fixed or random effects model with Review Manager (RevMan, the Cochrane Collaboration, Oxford, UK) version 5.3. According to the suggestions of the Cochrane collaboration tool's suggestions, Q statistics and the $\mathrm{I}^{2}$ index were used to assess heterogeneity, with significant heterogeneity indicated at $\mathrm{P}<0.05$ and an $\mathrm{I}^{2}-$ index $>50 \%$ (16). The estimates were pooled with a fixed effects model if no significant heterogeneity was identified, whereas a random effects model was used for estimates with heterogeneity.

\section{Results}

\section{Identification of eligible studies}

We identified 522 relevant studies, of which 157 duplicates were excluded. Another 345 studies were excluded due to irrelevant titles or abstracts. Seventeen studies were excluded as case reports, reviews, ongoing RCTs, no-control studies and conference abstracts. Finally, three studies were included in this study. One study was a RCT (15), and the remaining two were non-randomized studies $(17,18)$. The Preferred Reporting Items for Systematic Reviews and Meta-analyses (PRISMA) flow diagram (Figure 1) shows the entire review process from the original search to the final selection of studies. The RCT's data quality was assessed as "A" using Cochrane's collaboration tool for assessing the risk of bias. The Newcastle-Ottawa Scale score of the two non-randomized studies was 7 and 4 points for the Li et al. (17) and Kamiyama et al. (18) studies, respectively (Table 1).

\section{Randomized controlled trials}

\section{Patient characteristics}

A total of 164 HCC patients with PVTT were randomly and equally assigned to a neoadjuvant RT group and a hepatectomy alone group. Of these, most patients were classified as Eastern Cooperative Oncology Group (ECOG) 0 and Child-Pugh A, 16.4\% of patients had cirrhosis, and 92.1\% of patients were HBsAg positive. Most tumors were single, and Type II and III PVTT were the most common and were determined using Cheng's Classification. Specific details of the patient characteristics are recorded in Table 2.

\section{Treatment regimens}

Three-dimensional conformal RT (3DCRT) was delivered to the neoadjuvant RT group over a 5-day period, and the planned total dose to the planning target volume was 18 Gy, with a fractional size of 3.0 Gy. Hepatectomy was performed 4 weeks after neoadjuvant RT. 


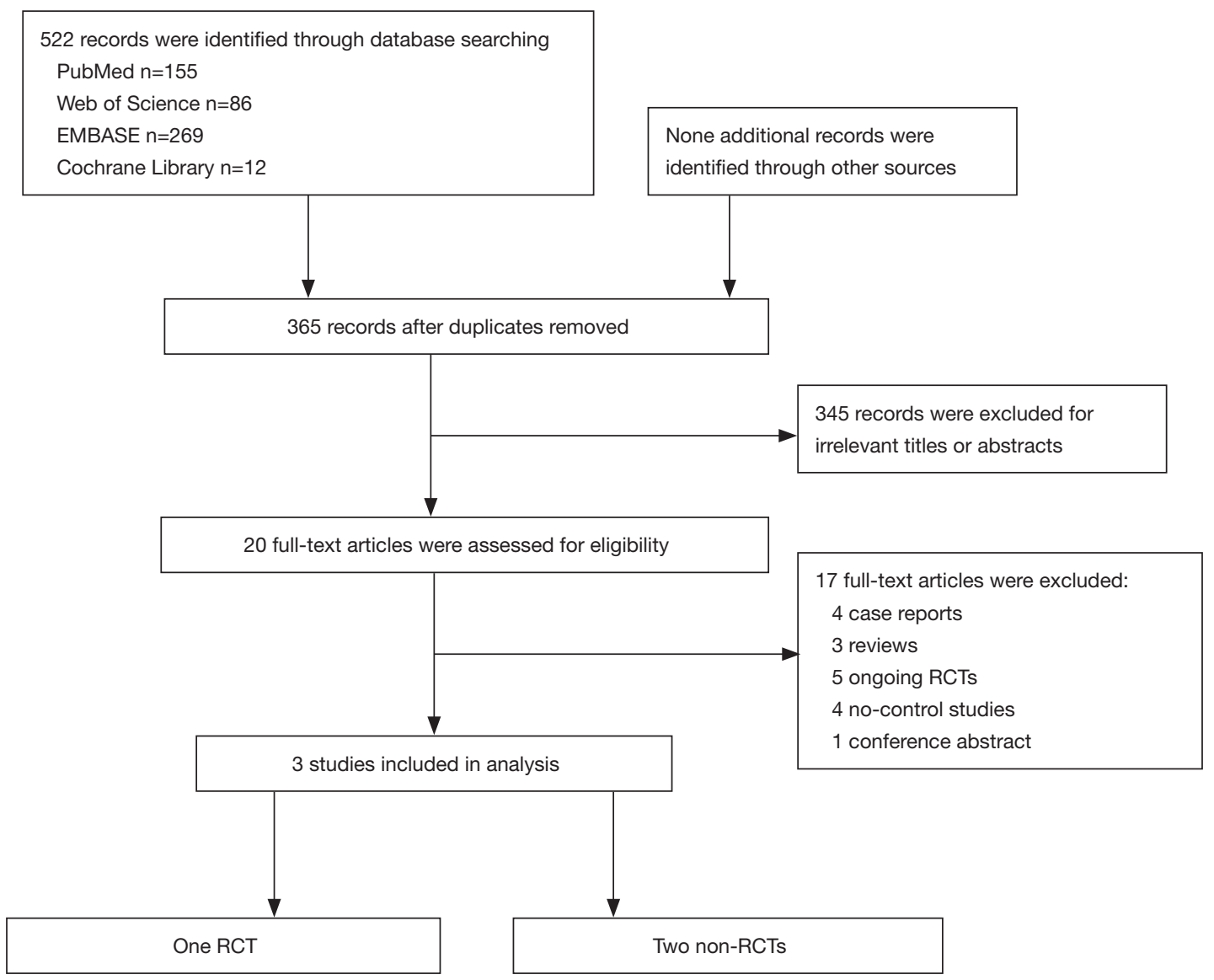

Figure 1 Search strategy for selection of studies. RCTs, randomized controlled trials.

\section{Non-randomized studies}

\section{Patient characteristics}

A total of 138 patients were included in the two studies, of whom 60 received neoadjuvant RT and 78 received surgery only. Nearly $80 \%$ of patients were $\mathrm{HBsAg}$ positive, $87.7 \%$ were Child-Pugh A, and 57.2\% [79] of patients had cirrhosis. The majority of patients $(74.6 \%)$ had a single tumor. Specific details of the patient characteristics are recorded in Table 2.

\section{Treatment regimens}

External RT or 3DCRT was delivered to the neoadjuvant RT group over a 6 to 12 -day period. The planned total dose to the planning target volume varied from 18 to $36 \mathrm{~Gy}$, with a fractional size of 3.0 Gy and the linear accelerator varied from 6 to 12 fractions. The interval between neoadjuvant
RT and hepatectomy varied from 2 to 4 weeks.

\section{RCTs}

\section{$\mathrm{RT}$ response rate}

Seventeen (20.7\%) patients were evaluated as partial response (PR) and had a positive RT response. Among the PR patients, 12 (70.6\%) had PVTT downstaged from Type III to Type II or from Type II to Type I. Also, 58 (70.7\%) patients had stable disease (SD) and seven (8.6\%) had progressive disease $(\mathrm{PD})$.

\section{Overall survival}

The median follow-up was 15.2 and 10.8 months in the neoadjuvant RT and surgery-alone groups, respectively. The 1-year OS rate in the neoadjuvant RT and surgeryalone groups was $75.2 \%$ and $43.1 \%(\mathrm{P}<0.001)$, respectively. 


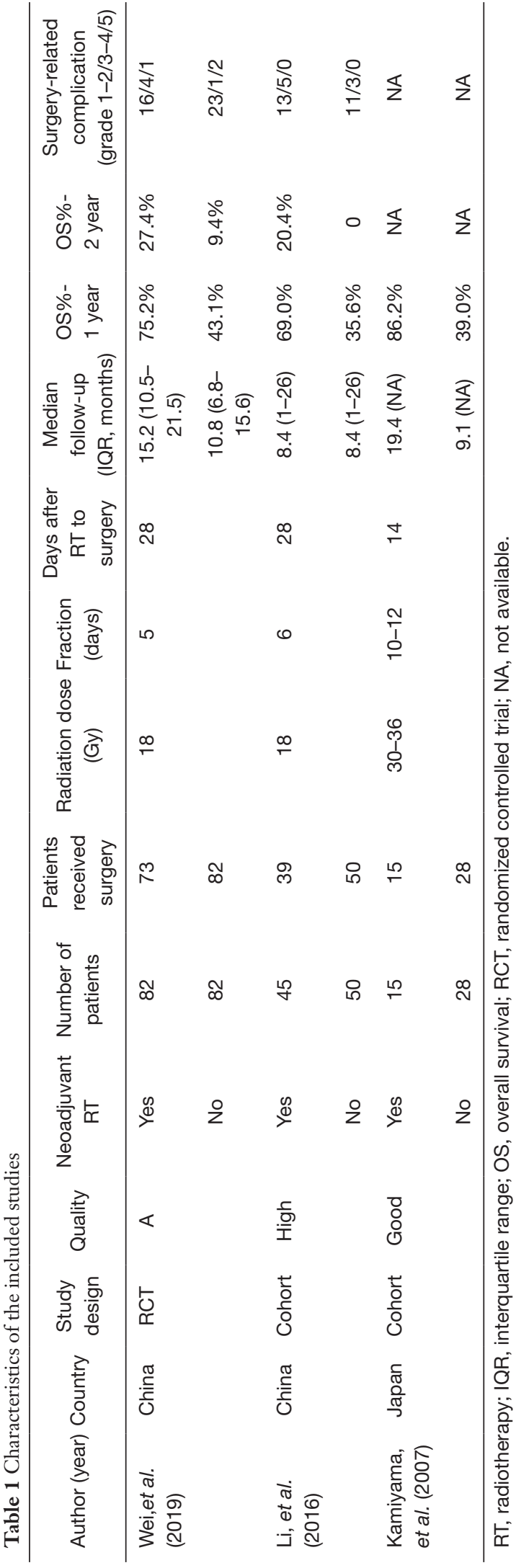

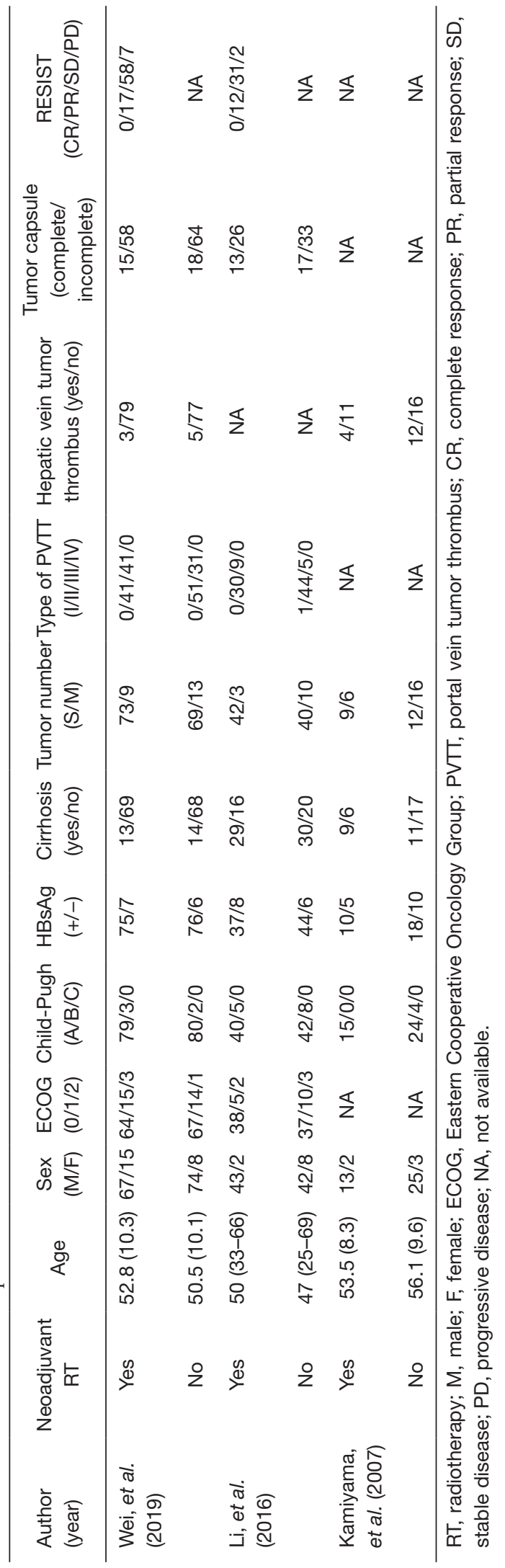




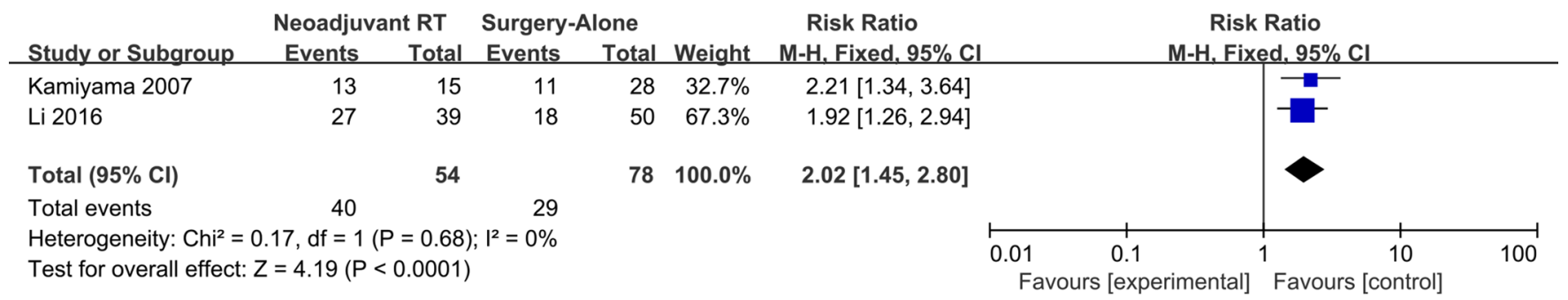

Figure 2 Effect of neoadjuvant radiotherapy on survival of patients with hepatocellular carcinoma and portal vein tumor thrombus in nonrandomized studies. Results of meta-analysis according to fixed model. CI, confidence interval.

The 2-year OS rate was $27.4 \%$ and $9.4 \%(\mathrm{P}<0.001)$ in the two groups.

\section{Complications}

After RT, seven patients had PD and two had liver toxicity. Therefore, they did not receive surgical treatment. In the neoadjuvant RT group, 21 patients had postoperative complications. Twenty-six patients had postoperative complications in the surgery-alone group.

\section{Non-RCTs}

\section{$\mathrm{RT}$ response rate}

In the Li et al. study, 12 (26.7\%) patients who were evaluated as PR had a positive RT response, 31 (68.9\%) had SD, and 2 (4.4\%) had PD.

\section{Overall survival}

Of the total $138 \mathrm{HCC}$ patients, the median follow-up ranged from 8.4 to 19.4 months for the neoadjuvant RT group and from 8.4 to 9.1 months for the surgery alone group. In the neoadjuvant RT group, the 1-year OS rate ranged from $69.0-86.2 \%$. In the surgery alone group, the 1 -year OS rate ranged from $35.6 \%$ to $39.0 \%$. The metaanalysis of the two included studies with a fixed effects model demonstrated a longer OS in patients who received neoadjuvant RT followed by hepatectomy compared with hepatectomy alone at 1 -year follow-up $(\mathrm{RR}=2.02$; $95 \% \mathrm{CI}$ : 1.45-2.80; $\mathrm{P}<0.0001$ ) (Figure 2).

\section{Complications}

After RT, six patients did not receive surgical treatment including two patients experienced a deterioration in liver function, and four had PD. In the neoadjuvant RT group, 18 patients had postoperative complications. In the hepatectomy alone group, 14 patients had postoperative complications.

\section{Discussion}

This systematic review included one randomized and two non-RCTs comparing combined neoadjuvant RT and surgery with surgery alone for HCC with PVTT. This review was based on the three studies with 302 patients and concluded that neoadjuvant RT was associated with longer survival, with results demonstrating 29 (22.8\%) patients were evaluated as partial response (PR) and had a positive RT response, but nine (7.1\%) had progressive disease (PD) after RT, a 1-year OS rate of $69.0-86.2 \%$ for patients receiving neoadjuvant $\mathrm{RT}$ and a 2 -year OS rate of more than $20 \%$.

PVTT commonly occurs in patients with advanced HCC and is found in approximately $10-40 \%$ of all HCC patients with a poor prognosis $(19,20)$. Mähringer-Kunz et al. reported that the median OS in patients without PVTT and with PVTT was 35.7 and 7.2 months, respectively, and they indicated that the extent of PVTT is not a decisive factor as even minor PVTT may lead to a dismal prognosis (21). The poor prognosis results from multiple factors including aggressive tumor behavior, deteriorated liver function reserve, limited treatment options, and high recurrence rates after treatment and PVTT can also facilitate the tumor spreading throughout the entire liver parenchyma (22). Zhang et al. established a scoring system for HCC with PVTT, which is calculated using total bilirubin, $\alpha$-fetoprotein (AFP), tumor diameter, and satellite lesions (23). This scoring system was used to assess whether patients were suitable for hepatectomy.

Traditionally, therapeutic options for HCC patients with PVTT are limited. Sorafenib was the only choice 
for those patients in the past. Recently, a study in The New England Fournal of Medicine reported that combined atezolizumab and bevacizumab, which had a 1-year OS rate of $67.2 \%$, significantly prolonged OS and PFS than sorafenib for unresectable HCC (24). In these patients, only $38 \%$ were macrovascular invasion but without clear PVTT types. It suggested that atezolizumab-bevacizumab could improve the OS for HCC with PVTT. Further studies about atezolizumab-bevacizumab and neoadjuvant RT are warranted to definite the two therapies applicable to different PVTT types. However, the Chinese Association of Liver Cancer's expert consensus demonstrated that surgical treatment is considered to be potentially curative and is the preferred treatment option for HCC patients with Type I and II PVTT (20). A multicenter study in Japan demonstrated that as long as the PVTT is limited to the first-order branch, surgery is associated with a longer survival outcome than non-surgical treatment, and the median survival time in the surgical group was 1.77 years longer than that in the non-surgical group in the ChildPugh A patients (25). Compared to TACE, HCC patients with PVTT undergoing surgery had better long-term survival outcomes, and the survival benefit conferred by hepatectomy was 7-33 months (11). Similarly, a metaanalysis concluded that surgical resection was related with better OS than TACE or other non-surgical treatment in HCC with PVTT, and the 1-year OS with surgery alone were $28-87 \%$ in the included studies and one study demonstrated that the 1-year OS of Type II and III PVTT after surgery were $51 \%$ and $36 \%$, respectively (26). Therefore, the OS after surgery alone in our review was similar to other studies, and the OS in the neoadjuvant RT group was higher than the surgery alone group and there was a $30-45 \%$ difference in 1-year survival with no significant difference in postoperative complications. However, hepatectomy is controversial to be used for patients with Type III PVTT patients. The expert consensus suggests that Type III PVTT patients can undergo surgery after tumor downstaging using RT and/or TACE (20).

3DCRT appeared to be an effective treatment for patients with HCC involving Type II and III PVTT, and the OS of the 3DCRT group was better than the surgery group (27). With a median follow-up of 41 months, a prospective multicenter study demonstrated that stereotactic body radiation therapy (SBRT) for patients with HCC is well-tolerated and is an effective treatment modality (28).
SBRT combined with TACE appeared to be more effective than SBRT alone, and the 1-year OS was $71.4 \%$ vs. $14.6 \%$, respectively (29). Further, Shui et al. demonstrated that SBRT used as the initial treatment for PVTT that is unsuitable for resection or TACE could achieve adequate thrombus shrinkage and portal vein flow restoration in the majority of cases (30). Therefore, it could offer patients an opportunity to undergo further treatment such as resection or TACE to increase survival time. Additionally, a study reported that HAIC's early response (ER) at four weeks showed that $33 \%$ patients had PR and $76.9 \%$ patients had downstaging of tumors of these PR patients (31). Compared to neoadjuvant RT in our review, the PR rate and downstaging rate of HAIC were higher, but the PD rate $(26 \%)$ was also higher. Neoadjuvant RT reduced the possibility of residual tumor or spread in the portal vein during surgery and contributed to the improved survival outcomes mainly by decreasing the tumor volume in PVTT, especially by downstaging the PVTT type (15). The radiation dose reported in our review was 18-36 Gy in 5-13 fractions, which was lower than SBRT for $\operatorname{HCC}(32,33)$. Previous studies have reported that high-dose 3DCRT is an effective treatment that provides a survival benefit $(34,35)$. The low dose for HCC with PVTT may decrease the risk of radiation-induced liver damage (RILD), which includes fatigue, weight gain, anicteric ascites, etc. (34). Therefore, low-dose neoadjuvant RT may be used to reduce RILD and tumor size to improve the safety and efficacy of surgery and contribute to patients' prolonged survival. However, only about $20 \%$ patients were evaluated as PR but the specific characteristics of these $\mathrm{PR}$ patients were unclear in our review. Patient with ECOG performance status $0-1$, ChildPugh A, single tumor and no hepatic vein tumor thrombus may be a good candidate for neoadjuvant RT, but still need further exploration. There are still some complications after neoadjuvant RT, as reported in our reviews, such as tumor progression, liver toxicity, and even deterioration in liver function, and these complications caused patients to lose the option of surgery.

There are several limitations of our study. First, the number of included articles is small. Second, all studies are from Asian countries, and the results of this meta-analysis might not apply to patients in Western countries. Third, as most of the studies were retrospective and non-randomized, there is a high chance of introducing selection bias in treatment choice. 


\section{Conclusions}

This systematic review concluded that neoadjuvant RT followed by hepatectomy in patients with resectable HCC and PVTT is associated with a longer OS than hepatectomy alone. These results provide evidence that may aid clinicians in making better decisions for selected patients. In the future, more prospective and multicenter studies evaluating survival outcomes are warranted.

\section{Acknowledgments}

We thank all the members of Multiple Disciplinary Team for Digestive System Cancer at Cancer Hospital, Chinese Academy of Medical Science.

Funding: This work was supported the Capital Health Research and Development of Special Fund Program (No. 2018-1-4021), the National Natural Science Foundation of China (No. 81672461), the Chinese Academy of Medical Sciences Innovation Fund for Medical Sciences (CIFMS) (Nos. 2016-I2M-1-001 and 2017-12M-4-002), Sanming Project of Medicine in Shenzhen (No. SZSM202011010).

\section{Footnote}

Reporting Checklist: The authors have completed the PRISMA reporting checklist. Available at https://hbsn. amegroups.com/article/view/10.21037/hbsn-20-854/rc

Conflicts of Interest: All authors have completed the ICMJE uniform disclosure form (available at https://hbsn. amegroups.com/article/view/10.21037/hbsn-20-854/coif). The authors have no conflicts of interest to declare.

Ethical Statement: The authors are accountable for all aspects of the work in ensuring that questions related to the accuracy or integrity of any part of the work are appropriately investigated and resolved.

Open Access Statement: This is an Open Access article distributed in accordance with the Creative Commons Attribution-NonCommercial-NoDerivs 4.0 International License (CC BY-NC-ND 4.0), which permits the noncommercial replication and distribution of the article with the strict proviso that no changes or edits are made and the original work is properly cited (including links to both the formal publication through the relevant DOI and the license). See: https://creativecommons.org/licenses/by-nc-nd/4.0/.

\section{References}

1. Bray F, Ferlay J, Soerjomataram I, et al. Global cancer statistics 2018: GLOBOCAN estimates of incidence and mortality worldwide for 36 cancers in 185 countries. CA Cancer J Clin 2018;68:394-424.

2. Villanueva A. Hepatocellular Carcinoma. N Engl J Med 2019;380:1450-62.

3. Yin J, Bo WT, Sun J, et al. New Evidence and Perspectives on the Management of Hepatocellular Carcinoma with Portal Vein Tumor Thrombus. J Clin Transl Hepatol 2017;5:169-76.

4. Forner A, Reig ME, de Lope CR, et al. Current strategy for staging and treatment: the BCLC update and future prospects. Semin Liver Dis 2010;30:61-74.

5. Heimbach JK, Kulik LM, Finn RS, et al. AASLD guidelines for the treatment of hepatocellular carcinoma. Hepatology 2018;67:358-80.

6. Zhao Y, Duran R, Chapiro J, et al. Transarterial Chemoembolization for the Treatment of AdvancedStage Hepatocellular Carcinoma. J Gastrointest Surg 2016;20:2002-9.

7. Liu M, Shi J, Mou T, et al. Systematic review of hepatic arterial infusion chemotherapy versus sorafenib in patients with hepatocellular carcinoma with portal vein tumor thrombosis. J Gastroenterol Hepatol 2020;35:1277-87.

8. Shi J, Lai EC, Li N, et al. Surgical treatment of hepatocellular carcinoma with portal vein tumor thrombus. Ann Surg Oncol 2010;17:2073-80.

9. Xu JF, Liu XY, Wang S, et al. Surgical treatment for hepatocellular carcinoma with portal vein tumor thrombus: a novel classification. World J Surg Oncol 2015;13:86.

10. Zhang XP, Wang K, Li N, et al. Survival benefit of hepatic resection versus transarterial chemoembolization for hepatocellular carcinoma with portal vein tumor thrombus: a systematic review and meta-analysis. BMC Cancer 2017;17:902.

11. Ibrahim C, Parra N, Macedo FI, et al. Is hepatic resection better than transarterial chemoembolization in hepatocellular carcinoma with portal vein tumor thrombosis? J Gastrointest Oncol 2019;10:1064-72.

12. Lee WH, Byun HK, Choi JS, et al. Liver-directed combined radiotherapy as a bridge to curative surgery in locally advanced hepatocellular carcinoma beyond the Milan criteria. Radiother Oncol 2020;152:1-7.

13. Sayan M, Yegya-Raman N, Greco SH, et al. Rethinking the Role of Radiation Therapy in the Treatment of Unresectable Hepatocellular Carcinoma: A Data Driven 
Treatment Algorithm for Optimizing Outcomes. Front Oncol 2019;9:345.

14. Im JH, Yoon SM, Park HC, et al. Radiotherapeutic strategies for hepatocellular carcinoma with portal vein tumour thrombosis in a hepatitis B endemic area. Liver Int 2017;37:90-100.

15. Wei X, Jiang Y, Zhang X, et al. Neoadjuvant ThreeDimensional Conformal Radiotherapy for Resectable Hepatocellular Carcinoma With Portal Vein Tumor Thrombus: a Randomized, Open-Label, Multicenter Controlled Study. J Clin Oncol 2019;37:2141-51.

16. Yeh SA, Chen YS, Perng DS. The role of radiotherapy in the treatment of hepatocellular carcinoma with portal vein tumor thrombus. J Radiat Res 2015;56:325-31.

17. Li N, Feng S, Xue J, et al. Hepatocellular carcinoma with main portal vein tumor thrombus: a comparative study comparing hepatectomy with or without neoadjuvant radiotherapy. HPB (Oxford) 2016;18:549-56.

18. Kamiyama T, Nakanishi K, Yokoo H, et al. Efficacy of preoperative radiotherapy to portal vein tumor thrombus in the main trunk or first branch in patients with hepatocellular carcinoma. Int J Clin Oncol 2007;12:363-8.

19. Wang JC, Xia AL, Xu Y, et al. Comprehensive treatments for hepatocellular carcinoma with portal vein tumor thrombosis. J Cell Physiol 2019;234:1062-70.

20. Cheng S, Chen M, Cai J, et al. Chinese Expert Consensus on Multidisciplinary Diagnosis and Treatment of Hepatocellular Carcinoma with Portal Vein Tumor Thrombus (2018 Edition). Liver Cancer 2020;9:28-40.

21. Mähringer-Kunz A, Steinle V, Duber C, et al. Extent of portal vein tumour thrombosis in patients with hepatocellular carcinoma: The more, the worse? Liver Int 2019;39:324-31.

22. Liu PH, Huo TI, Miksad RA. Hepatocellular Carcinoma with Portal Vein Tumor Involvement: Best Management Strategies. Semin Liver Dis 2018;38:242-51.

23. Zhang XP, Gao YZ, Chen ZH, et al. An Eastern Hepatobiliary Surgery Hospital/Portal Vein Tumor Thrombus Scoring System as an Aid to Decision Making on Hepatectomy for Hepatocellular Carcinoma Patients With Portal Vein Tumor Thrombus: A Multicenter Study. Hepatology 2019;69:2076-90.

24. Finn RS, Qin S, Ikeda M, et al. Atezolizumab plus Bevacizumab in Unresectable Hepatocellular Carcinoma. N Engl J Med 2020;382:1894-905.

25. Kokudo T, Hasegawa K, Matsuyama Y, et al. Survival benefit of liver resection for hepatocellular carcinoma associated with portal vein invasion. J Hepatol
2016;65:938-43.

26. Liang L, Chen TH, Li C, et al. A systematic review comparing outcomes of surgical resection and non-surgical treatments for patients with hepatocellular carcinoma and portal vein tumor thrombus. HPB (Oxford) 2018;20:1119-29.

27. Su F, Chen KH, Liang ZG, et al. Comparison of threedimensional conformal radiotherapy and hepatic resection in hepatocellular carcinoma with portal vein tumor thrombus. Cancer Med 2018;7:4387-95.

28. Jang WI, Bae SH, Kim MS, et al. A phase 2 multicenter study of stereotactic body radiotherapy for hepatocellular carcinoma: Safety and efficacy. Cancer 2020;126:363-72.

29. Choi HS, Kang KM, Jeong BK, et al. Effectiveness of stereotactic body radiotherapy for portal vein tumor thrombosis in patients with hepatocellular carcinoma and underlying chronic liver disease. Asia Pac J Clin Oncol 2021;17:209-15.

30. Shui Y, Yu W, Ren X, et al. Stereotactic body radiotherapy based treatment for hepatocellular carcinoma with extensive portal vein tumor thrombosis. Radiat Oncol 2018;13:188.

31. Lin CC, Hung CF, Chen WT, et al. Hepatic Arterial Infusion Chemotherapy for Advanced Hepatocellular Carcinoma with Portal Vein Thrombosis: Impact of Early Response to 4 Weeks of Treatment. Liver Cancer 2015;4:228-40.

32. Andolino DL, Johnson CS, Maluccio M, et al. Stereotactic body radiotherapy for primary hepatocellular carcinoma. Int J Radiat Oncol Biol Phys 2011;81:e447-53.

33. Bujold A, Massey CA, Kim JJ, et al. Sequential phase I and II trials of stereotactic body radiotherapy for locally advanced hepatocellular carcinoma. J Clin Oncol 2013;31:1631-9.

34. Hou JZ, Zeng ZC, Wang BL, et al. High dose radiotherapy with image-guided hypo-IMRT for hepatocellular carcinoma with portal vein and/or inferior vena cava tumor thrombi is more feasible and efficacious than conventional 3D-CRT. Jpn J Clin Oncol 2016;46:357-62.

35. Rim CH, Yang DS, Park YJ, et al. Effectiveness of highdose three-dimensional conformal radiotherapy in hepatocellular carcinoma with portal vein thrombosis. Jpn J Clin Oncol 2012;42:721-9.

Cite this article as: Wei Z, Zhao J, Bi X, Zhang Y, Zhou J, Li Z, Huang Z, Zhao H, Cai J. Neoadjuvant radiotherapy for resectable hepatocellular carcinoma with portal vein tumor thrombus: a systematic review. HepatoBiliary Surg Nutr 2022;11(5):709-717. doi: 10.21037/hbsn-20-854 